\title{
Realizing the Distance Matrix of a Graph
}

\author{
A. J. Goldman \\ Institute for Basic Standards, National Bureau of Standards, Washington, D.C. \\ (February 23, 1966)

\begin{abstract}
An explicit description is given for the unique graph with as few arcs (each bearing a positive length) as possible, which has a prescribed matrix of shortest-path distances between pairs of distinct vertices. The same is done in the case when the $i$ th diagonal matrix entry, instead of being zero, represents the length of a shortest closed path containing the $i$ th vertex.
\end{abstract}

Key Words: Graph, distance matrix, shortest path.

Let $G$ be a finite oriented graph with vertices $\left\{v_{i}\right\}_{1}^{n}$, where $n>2$. To avoid unnecessary complications, we restrict attention to connected graphs, i.e., if $i \neq j$ then $G$ contains a directed path from $v_{i}$ to $v_{j}$. As additional structure, we assume associated to $G$ a positive-valued function $l_{G}$ assigning lengths $l_{G}(i, j)$ to the $\operatorname{arcs}\left(v_{i}, v_{j}\right)$ of $G$.

The distance matrix $D_{G}$ of $G$ has entries $d_{G}(i, i)=0$ on the main diagonal; a typical off-diagonal entry $d_{G}(i, j)$ repersents the length of a shortest directed path in $G$ from $v_{i}$ to $v_{j}$. An arc of $G$ is called redundant if its deletion leaves $D_{G}$ unchanged. The graph $G$ will be called irreducible if it contains no redundant arcs.

A real square matrix $D$ with entries $d(i, j)$ is called realizable if there is a graph $G$ such that $D=D_{G}$. Hakimi and $\mathrm{Yau}^{1}$ showed that necessary and sufficient conditions for the realizability of $D$ are

$$
\begin{aligned}
& d(i, i)=0, \\
& d(i, j)>0 \quad \text { if } i \neq j, \\
& d(i, j) \leqslant d(i, k)+d(k, j) .
\end{aligned}
$$

The necessity of these conditions should be clear. To prove sufficiency one need only take the ares of $G$ to be all $\left(v_{i}, v_{j}\right)$ with $i \neq j$, and define $l_{G}$ by $l_{G}(i, j)=d(i, j)$; it follows readily from (1) to (3) that $D_{G}=D$.

If matrix $D$ is realizable, it clearly has a realization by an irreducible graph. Hakimi and Yau (op. cit.) showed that this irreducible representation was unique, but did not give an explicit description of it. Our first purpose in this note is to provide such a description.

THEOREM 1. Let $\mathrm{G}$ be an irreducible representation of $\mathrm{D} . \operatorname{Arc}\left(\mathbf{v}_{\mathbf{i}}, \mathbf{v}_{\mathbf{j}}\right)$ is present in $\mathrm{G}$ if and only if $\mathrm{i} \neq \mathrm{j}$ and

$$
d(i, j)<\min \{d(i, k)+d(k, j): k \neq i, j\} .
$$

${ }^{1}$ S. L. Hakimi and S. S. Yau, Distance matrix of a graph and its realizability, Q. Applied Math., Jan. 1965, 305-317.
In this case,

$$
l_{G}(i, j)=d(i, j) .
$$

We remark that it follows that $G$ can be constructed (simultaneously with the checking of (3)) in the following way. Replace the zeros on the main diagonal of $D$ by $\infty$, obtaining a new matrix $E=\left(e_{i j}\right)$. Form $E^{2}$ $=\left(e_{i j}^{(2)}\right)$ using the special "matrix multiplication" often employed for shortest-path problems, i.e.,

$$
e_{i j}^{(2)}=\min _{k}\left(e_{i k}+e_{k j}\right) .
$$

(D. Rosenblatt has pointed out the relation of this operation to the Peirce-Schroder relative sum; see e.g., B. Russell's "Principles of Mathematics.") In view of (3) and (4), arc $\left(v_{i}, v_{j}\right)$ is present in $G$ if and only if $i \neq j$ and $e_{i j} \neq e_{i j}^{(2)}$; if present, its length is given by (5).

We begin the proof by observing that $G$, because of its irreducibility, contains no ares of the form $\left(v_{i}, v_{i}\right)$. Thus arc $\left(v_{i}, v_{j}\right)$ can be present in $G$ only if $i \neq j$.

If $\operatorname{arc}\left(v_{i}, v_{j}\right)$ is present in $G$, it constitutes a path from $v_{i}$ to $v_{j}$, and so

$$
l_{G}(i, j) \geqslant d_{G}(i, j)=d(i, j) .
$$

If strict inequality held in (6), then there would be a shortest path $P_{i j}$ from $v_{i}$ to $v_{j}$ (in $G$ ) which does not contain $\left(v_{i}, v_{j}\right)$, and no path of $G$ would be lengthened if each appearance of $\left(v_{i}, v_{j}\right)$ in it were replaced by $P_{i j}$. Therefore $\left(v_{i}, v_{j}\right)$ would be redundant, a contradiction. So (5) is proved.

Suppose (4) does not hold, i.e., there is a $k \neq i, j$ such that

$d_{G}(i, j)=d(i, j) \geqslant d(i, k)+d(k, j)=d_{G}(i, k)+d_{G}(k, j)$.

Let $P_{i k}$ be a shortest path in $G$ from $v_{i}$ to $v_{k}, P_{k j}$ a shortest path from $v_{k}$ to $v_{j}$, and $Q_{i j}$ the composition of $P_{i k}$ and $P_{k j}$. If arc $\left(v_{i}, v_{j}\right)$ were present in $G$, then by (2), (5) and (7) it could not lie in $P_{i k}$ or $P_{k j}$, and hence not in $Q_{i j}$. It follows from (5) and (7) that no path in 
$G$ would be lengthened if each appearance of $\left(v_{i}, v_{j}\right)$ in it were replaced by $Q_{i j}$. Thus $\left(v_{i}, v_{j}\right)$ would be redundant, a contradiction. We have proved that (4) is a necessary condition for the presence of $\left(v_{i}, v_{j}\right)$ in $G$.

It only remains to rule out the possibility that (4) holds but arc $\left(v_{i}, v_{j}\right)$ is absent from $G$. Let the successive vertices of a shortest path in $G$ from $v_{i}$ to $v_{j}$ be $v_{i}, v_{k(1)}, \ldots, v_{k(m)}, v_{j}$ where $m \geqslant 1$ (because $\left(v_{i}, v_{j}\right)$ is absent). Then by (5),

$$
d(i, j)=d_{G}(i, j)=d(i, k(1))+\ldots+d(k(m), j) .
$$

Repeated application of the triangle inequality (3) to the sum in (8) yields

$$
d(i, j) \geqslant d(i, k(1))+d(k(1), j),
$$

contradicting (4). This completes the proof of the theorem.

CoRollary. A graph $\mathrm{G}$ is irreducible if and only if, for each of its arcs $\left(\mathbf{v}_{\mathbf{i}}, \mathbf{v}_{\mathbf{j}}\right)$,

$$
\mathrm{l}_{\mathrm{G}}(\mathrm{i}, \mathrm{j})=\mathrm{d}_{\mathrm{G}}(\mathrm{i}, \mathrm{j})<\min \left\{\mathrm{d}_{\mathrm{G}}(\mathrm{i}, \mathrm{k})+\mathrm{d}_{\mathrm{G}}(\mathrm{k}, \mathrm{j}): \mathrm{k} \neq \mathrm{i}, \mathrm{j}\right\} .
$$

We pass now to a second type of "distance matrix," denoted $D_{G}^{*}=\left(d^{*}(i, j)\right)$, obtained from $D$ by changing the main diagonal's entries from $d_{G}(i, i)=0$ to $d_{G}^{*}(i, i)$, the length of a shortest closed path of $G$ which contains $v_{i}$.

Theorem 2. A matrix $\mathrm{D}^{*}$ is realizable as a $\mathrm{D}_{\mathrm{G}}^{*}$ if and only if its entries $\mathrm{d}^{*}(\mathrm{i}, \mathrm{j})$ satisfy

$$
\begin{aligned}
& d^{*}(i, j)>0 \\
& d^{*}(i, j) \leqslant d^{*}(i, k)+d^{*}(k, j), \\
& d^{*}(i, i) \leqslant \min \left\{d^{*}(i, j)+d^{*}(j, i): j \neq i\right\} .
\end{aligned}
$$

The necessity of (10) and (11) should be clear. As for (12), observe that any closed path $C$ of $G$ containing $v_{i}$ and at least one other vertex can be regarded as consisting of a path from $v_{i}$ to some other vertex $v_{j}$ of $C$, followed by a path from $v_{j}$ back to $v_{i}$; the minimum possible lengths of these two paths are $d_{G}^{*}(i, j)$ and $d_{G}^{*}(j, i)$, respectively. Thus the shortest closed path containing $v_{i}$ and $v_{j}(j \neq i)$ has length $d_{G}^{*}(i, j)$ $+d_{G}^{*}(j, i)$, and so

$$
d_{G}^{*}(i, i) \leqslant \min \left\{d_{G}^{*}(i, j)+d_{G}^{*}(j, i): j \neq i\right\},
$$

from which the necessity of (12) follows. Note that equality holds in (13) unless $\left(v_{i}, v_{i}\right) \in G$. (If our definition of "graph" were restricted to exclude arcs of the form $\left(v_{i}, v_{i}\right)$, then equality would hold in (12).)

The sufficiency proof is as for $D_{G}$, except that the realizing graph is given an arc $\left(v_{i}, v_{i}\right)$ for each index $i$ such that strict inequality holds in (12).

We now assume the definitions of "redundant" and "irreducible" modified to apply to $D_{G}^{*}$ rather than $D_{G}$. The analog of theorem 1 requires no new arguments. The conclusion is that if $D^{*}$ can be realized as a $D_{G}^{*}$, it has a unique irreducible realization, whose graph $G$ is found as follows. Change the main diagonal terms of $D^{*}$ to zero, obtaining a matrix $D$ satisfying (1) to (3). We have previously described how to construct the unique irreducible graph $H$ such that $D_{H}=D$. Adjoin to $H$ an $\operatorname{arc}\left(v_{i}, v_{i}\right)$ for each $i$ such that strict inequality holds in (12), and let the length of this arc be $d^{*}(i, i)$.

(Paper 70B2-176) 